\title{
Differential expression of laminin isoforms in diabetic nephropathy and other renal diseases
}

\author{
Suman Setty ${ }^{1}$, Alfred A Michael ${ }^{2}$, Alfred J Fish ${ }^{2}$, S Michael Mauer ${ }^{2}$, Ralph J Butkowski², \\ Ismo Virtanen $^{3}$ and Youngki Kim ${ }^{2}$ \\ ${ }^{1}$ Department of Pathology, University of Illinois at Chicago, Chicago, IL, USA; ${ }^{2}$ Department of Pediatric \\ Nephrology, University of Minnesota Medical School, Minneapolis, MN, USA and ${ }^{3}$ Institute of Biomedicine/ \\ Anatomy, University of Helsinki, Helsinki, Finland
}

\begin{abstract}
Laminin a non-collagenous glycoprotein is a major component of the renal glomerular basement membrane and mesangium. Thus far eleven distinct chains have been described, permutations of which make up 15 laminin isoforms. Laminin molecules interact with cells and other matrix molecules during organ development and differentiation. We studied the distribution of laminin isoforms in patients with type 1 diabetic nephropathy, membranous nephropathy, membranoproliferative glomerulonephritis and IgA nephropathy/ Henoch-Schönlein purpura. Immunofluorescence microscopic studies with laminin-chain-specific antibodies to the $\alpha 1, \alpha 2$, $\alpha 5, \beta 1, \beta 2$ and $\gamma 1$ chains detected $\alpha 2, \beta 1$ and $\gamma 1$ chain expression in the normal mesangium and $\alpha 5, \beta 2$ and $\gamma 1$ in normal glomerular basement membrane. Significantly, constituents of the glomerular basement membrane, $\alpha 5$, $\beta 2$ and $\gamma 1$ chains were overexpressed in kidneys with diabetic nephropathy. Initially the constituents of the mesangium increased commensurate with the degree of mesangial expansion and degree of diabetic nephropathy. Reduction in $\alpha 2$ chain intensity was observed with severe mesangial expansion and in the areas of nodular glomerulosclerosis. In addition, with late disease aberrant expression of $\alpha 2$ and $\beta 2$ chains was observed in the mesangium. Glomerular basement membrane in renal disease overexpressed molecules normally present in that location. In summary, the alterations in basement membrane composition in various renal diseases seem to not only reflect the balance between synthesis and degradation of normal basement membrane constituents, but also their aberrant expression.

Modern Pathology (2012) 25, 859-868; doi:10.1038/modpathol.2011.216; published online 17 February 2012
\end{abstract}

Keywords: diabetic nephropathy; isoforms; laminin

Laminin is a major non-collagenous glycoprotein component of glomerular basement membrane and mesangium and consists of three different polypeptide chains designated $\alpha, \beta$ and $\gamma$. Laminin was first isolated from EHS mouse tumor and subsequently found to be a cross-shaped disulfide-bonded heterotrimer composed of a $400-\mathrm{kDa} \alpha 1$ chain along with $\beta 1$ and $\gamma 1$ chains of approximately $200 \mathrm{kDa}$. Eleven distinct laminin chains have been described to date (five $\alpha$, three $\beta$ and three $\gamma$ chains). These chains can combine into at least 15 different laminin isoforms, Lm-111 to Lm-523. ${ }^{1-3}$

Correspondence: Dr S Setty, MD, PhD, Department of Pathology (MC 847), College of Medicine, 840 S. Wood St., Room 130, Chicago, IL 60612, USA.

E-mail: ssetty@uic.edu

Received 14 May 2009; revised 25 November 2011; accepted 9

December 2011; published online 17 February 2012
Laminins are involved in multiple interactions with other basement membrane components and cells and play important roles in the supramolecular assembly of extracellular matrix and the stability of basement membrane. ${ }^{3-5}$ Laminins also help mediate various cellular functions, including growth, differentiation and attachment. The distribution of laminin chains in the glomerulus is site-specific and associated with the presence of different isoforms in the glomerular basement membrane and the mesangium. Therefore the $\alpha 5, \beta 2$ and $\gamma 1$ chains are present in normal adult human glomerular basement membrane, whereas $\alpha 1, \alpha 2$ and $\beta 1$ chains are not. ${ }^{2,6-8}$ Only $\alpha 2, \alpha 4, \beta 1$ and $\gamma 1$ chains are present in the normal adult mesangium. ${ }^{2,9}$ Therefore, glomerular basement membrane and mesangium in normal adult human kidneys expresses different isoforms of laminin: Lm-521 ( $\alpha 5, \beta 2$ and $\gamma 1)$ in glomerular basement membrane and Lm-211/411 ( $\alpha 2 / \alpha 4, \quad \beta 1$ and $\gamma 1$ ) in the mesangium. 
In addition, there is considerable variation in the distribution of laminin isoforms in the developing kidney. Nephrogenesis is a complex process during which the unique elements of the glomerular basement membrane, mesangial matrix, tubular basement membrane and blood vessels come together. In developing rodent glomeruli, the transition of type IV collagen isoforms is accompanied by the transition of laminin isoforms. ${ }^{2,10}$ Although collagen $\alpha 1$ (IV) and $\alpha 2$ (IV) are found in all developing

Table 1 Specificity and source of antibodies

\begin{tabular}{llll}
\hline Antibodies & Specificity & Source & References \\
\hline 161EB7 & Laminin $\alpha 1$ & I Virtanen & 8 \\
2G9 & Laminin $\alpha 2$ & E Engvall/Chemicon & 30 \\
5H2/H7 & Laminin $\alpha 2$ & E Engvall & 30 \\
4C7 & Laminin $\alpha 5$ & E Engvall/Chemicon & 31 \\
3E5 & Laminin $\beta 1$ & E Engvall/Chemicon & 31 \\
C4 & Laminin $\beta 2$ & JR Sanes/Hybridoma Bank & 32 \\
D5 & Laminin $\beta 2$ & JR Sanes/Hybridoma Bank & 32 \\
2E8 & Laminin $\gamma 1$ & Hybridoma Bank & \\
102 & $\alpha 1($ IV)NC & University of MN laboratory & 33
\end{tabular}

Abbreviation: $\alpha 1$ (IV)NC $=$ non-collagenous domain of $\alpha 1$ chain of type IV collagen. glomerular basement membrane together with laminin $\beta 1$ chain, the expression of laminin $\beta 2$ chain is associated with collagen $\alpha 3-5$ (IV) in the glomerular

Table 2 Laminin chain distribution in human kidneys

\begin{tabular}{|c|c|c|c|c|c|c|}
\hline Antibody to & $\alpha 1$ & $\alpha 2$ & $\alpha 5$ & $\beta 1$ & $\beta 2$ & $\gamma 1$ \\
\hline \multicolumn{7}{|l|}{$G B M$} \\
\hline Normal & - & - & + & - & + & + \\
\hline Diabetic nephropathy & - & - & $\uparrow$ & - & $\uparrow$ & $\uparrow$ \\
\hline Membranous nephropathy & - & - & $\uparrow$ & - & $\uparrow$ & $\uparrow$ \\
\hline $\begin{array}{l}\text { Membranoproliferative } \\
\text { glomerulonephritis }\end{array}$ & - & - & $\rightarrow$ & - & $\rightarrow$ & $\rightarrow$ \\
\hline $\begin{array}{l}\text { Henoch-Schönlein } \\
\text { purpura/IgA nephropathy }\end{array}$ & - & - & $\rightarrow$ & - & $\rightarrow$ & $\rightarrow$ \\
\hline \multicolumn{7}{|l|}{ Mesangium } \\
\hline Normal & - & + & - & + & - & + \\
\hline Mild diabetic nephropathy & - & $\uparrow \uparrow$ & $\uparrow$ & $\uparrow \uparrow$ & $\uparrow$ & $\uparrow \uparrow$ \\
\hline Severe diabetic nephropathy & - & $\downarrow /-$ & $\uparrow$ & $\uparrow \uparrow$ & $\uparrow$ & $\uparrow \uparrow$ \\
\hline $\begin{array}{l}\text { Membranoproliferative } \\
\text { glomerulonephritis }\end{array}$ & - & $\downarrow /-$ & $\uparrow$ & $\uparrow \uparrow$ & $\uparrow$ & $\uparrow \uparrow$ \\
\hline $\begin{array}{l}\text { Henoch-Schönlein } \\
\text { purpura/IgA nephropathy }\end{array}$ & - & $\uparrow \uparrow$ & $\uparrow$ & $\uparrow \uparrow$ & $\uparrow$ & $\uparrow \uparrow$ \\
\hline Membanous nephropathy & - & $\rightarrow / \uparrow$ & $\rightarrow / \uparrow$ & $\rightarrow / \uparrow$ & $\rightarrow / \uparrow$ & $\rightarrow / \uparrow$ \\
\hline
\end{tabular}

- absent; + present; $\uparrow$ increase; $\downarrow$ decrease; $\rightarrow$ no change.
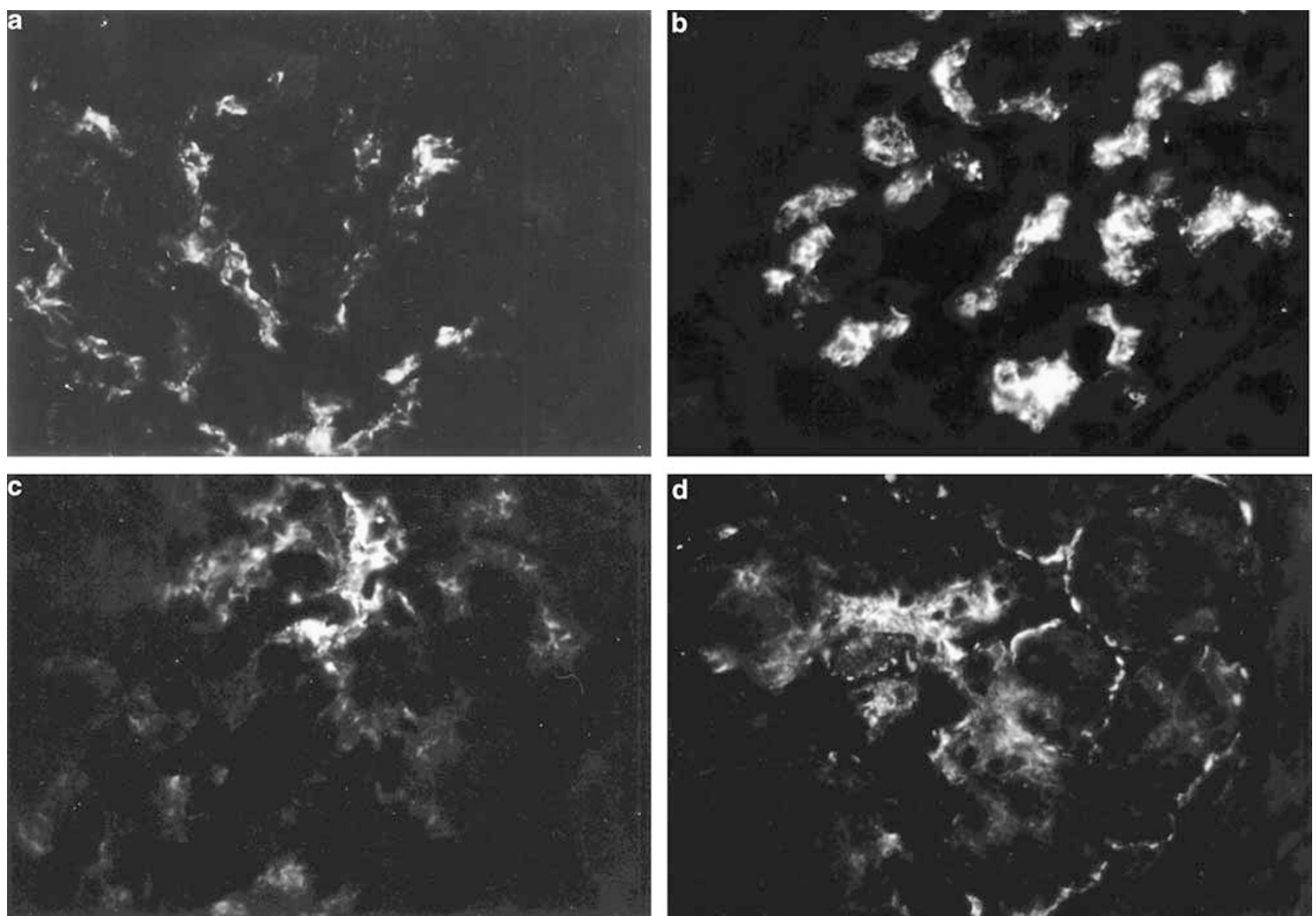

Figure 1 Immunofluorescence microscopy of glomeruli immunoreacted for $\operatorname{Lm} \alpha 2$ chain $(\times 550)$. (a) Normal kidney; (b) mild diabetic nephropathy; (c) severe diabetic nephropathy; (d) membranoproliferative glomerulonephritis. In the normal glomerulus, there is a variable immunoreaction in the mesangium. In mild diabetic nephropathy, there is a diffuse increase in mesangial staining. In contrast, note the decrease to disappearance of mesangial staining in severe diabetic nephropathy and membranoproliferative glomerulonephritis. 
basement membrane of more mature glomeruli. In other words, transition of collagen $\alpha 1-2$ (IV) to $\alpha 3-5$ (IV) during the glomerular basement membrane development is associated with the transition of laminin chains from $\beta 1$ to $\beta 2$. Mutant mice lacking the COL4A3 gene for $\alpha 3$ (IV) also lack $\alpha 3-5$ (IV) chains in the glomerular basement membrane with persistence of $\alpha 1-2$ (IV). ${ }^{11}$ Nonetheless, the laminin $\beta 1$ to $\beta 2$ switch occurs in rodents ${ }^{11}$ and in man. ${ }^{6}$ This suggests that collagen IV and laminin networks in glomerular basement membrane are regulated independently. Mutant mice lacking laminin $\beta 2$ chain show structurally intact glomerular basement membrane $\mathrm{M}$ with normal developmental transition of type IV collagen isoforms. ${ }^{12}$ The severe proteinuria in these mice suggests that the laminin $\beta 2$ chain has an important role in glomerular permselectivity through the maintenance of normal glomerular basement membrane structure or normal podocyte maturation. Recent studies show that glomerular basement membrane is a real barrier for albumin as proteinuria precedes podocyte abnormality in Lam $\beta 2^{-1 /-1}$ mice. ${ }^{13}$ Taken together, the transitions of laminin and type IV collagen isoforms, which normally coincide, appear to have specific roles in various stages of renal development. In this report, we examined the distribution of laminin isoforms in normal human kidneys and in various disease states.

\section{Materials and methods}

Kidney tissues were obtained from 12 patients with overt type 1 diabetic nephropathy, 5 patients with type I idiopathic membranoproliferative glomerulonephritis, 12 patients with Henoch-Schönlein purpura/IgA nephropathy, 5 patients with idiopathic membranous nephropathy and 14 normal controls including three adult kidney donors (age range, 3 to 59 years). The frozen material used in this study was residue of biopsies obtained for diagnostic purposes. This study was performed with the permission of the Institutional Review Board of the University of Minnesota.

\section{Antibodies}

The monoclonal antibodies used in this study are listed in Table 1. FITC-conjugated and
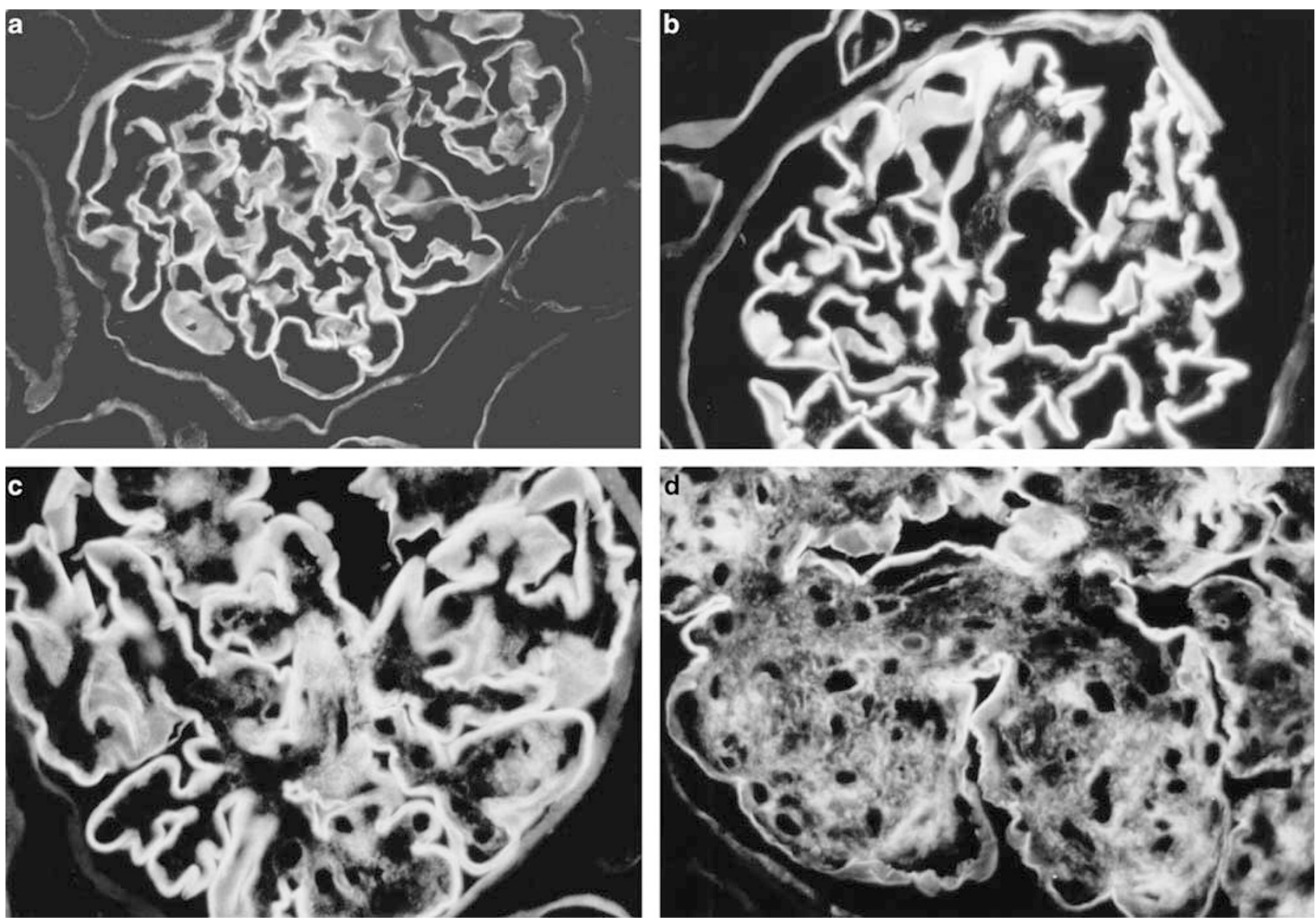

Figure 2 Immunofluorescence microscopy of glomeruli immunoreacted for Lm $\alpha 5$ chain $(\times 550)$. (a) Normal kidney; (b) mild diabetic nephropathy; (c) severe diabetic nephropathy; (d) membranoproliferative glomerulonephritis. In the normal glomerulus the Lm $\alpha 5$ chain overlies the glomerular basement membrane but is not detected in the mesangium. In diabetic nephropathy the Lm $\alpha 5$ chain overlies the thickened glomerular basement membrane. There is only a segmental reaction in the mesangium in mild diabetic nephropathy, which progresses to diffuse staining as the mesangium expands further in severe diabetic nephropathy. In membranoproliferative glomerulonephritis, the $\mathrm{Lm} \alpha 5$ chain is detected in the expanded mesangium and glomerular basement membrane. 
tetramethylrhodamine isothiocyanate (RITC)-conjugated goat anti-mouse IgG were obtained from Caltag (South San Francisco, CA, USA) and ICN-Cappel (Irvine, CA, USA); FITC-conjugated goat anti-rat IgG from Pel Freeze (Rogers, AR, USA) and Jackson Laboratories (West Grove, PA, USA); FITC-conjugated goat anti-human IgG from Kallestedt (Austin, TX, USA) and ICN-Cappel. All secondary antibodies were absorbed with normal human serum.

\section{Immunohistological Methods}

Immunofluorescence studies were carried out as previously described. ${ }^{14}$ Kidney tissues were snapfrozen in isopentane precooled in liquid nitrogen, sectioned at $4 \mu \mathrm{m}$ in a cryostat at constant room temperature $\left(25^{\circ} \mathrm{C}\right)$ and humidity $(30 \%)$, and fixed with acetone for $10 \mathrm{~min}$. The slides were exposed to primary antibodies followed by FITC-conjugated secondary antibodies. Dual fluorochrome labeling was performed using mouse monoclonal anti-laminin $\alpha 2$ chain detected with rhodamine-conjugated goat anti-mouse IgG, followed by rat monoclonal anti-type IV collagen $(\alpha 1$ (IV)NC, where NC denotes non-collagenous) detected with FITC-conjugated goat anti-rat IgG (absorbed with human and mouse sera). Appropriate controls were as previously described. ${ }^{14}$ P-phenylenediamine in PBS glycerol was applied to fluorochrome-stained tissue sections to retard fluorescence quenching and sections were examined using epifluoroscence microscope with appropriate filters (Carl Zeiss, Oberkochen, Germany).

\section{Morphometry}

Routine stereological techniques, previously described in detail, ${ }^{15}$ were used to measure mesangial fraction of glomerular volume in 12 patients with diabetic nephropathy. Mesangial fraction of glomerular volume in patients with diabetic nephropathy ranged from 0.22 to 0.55 with normal being $<0.2$.

\section{Results}

Immunoreactivity for $\operatorname{Lm} \alpha 5, \quad \beta 2$ and $\gamma 1$ chains was found in the glomerular basement membrane in normal adult human kidneys, whereas $\operatorname{Lm} \alpha 1, \alpha 2$
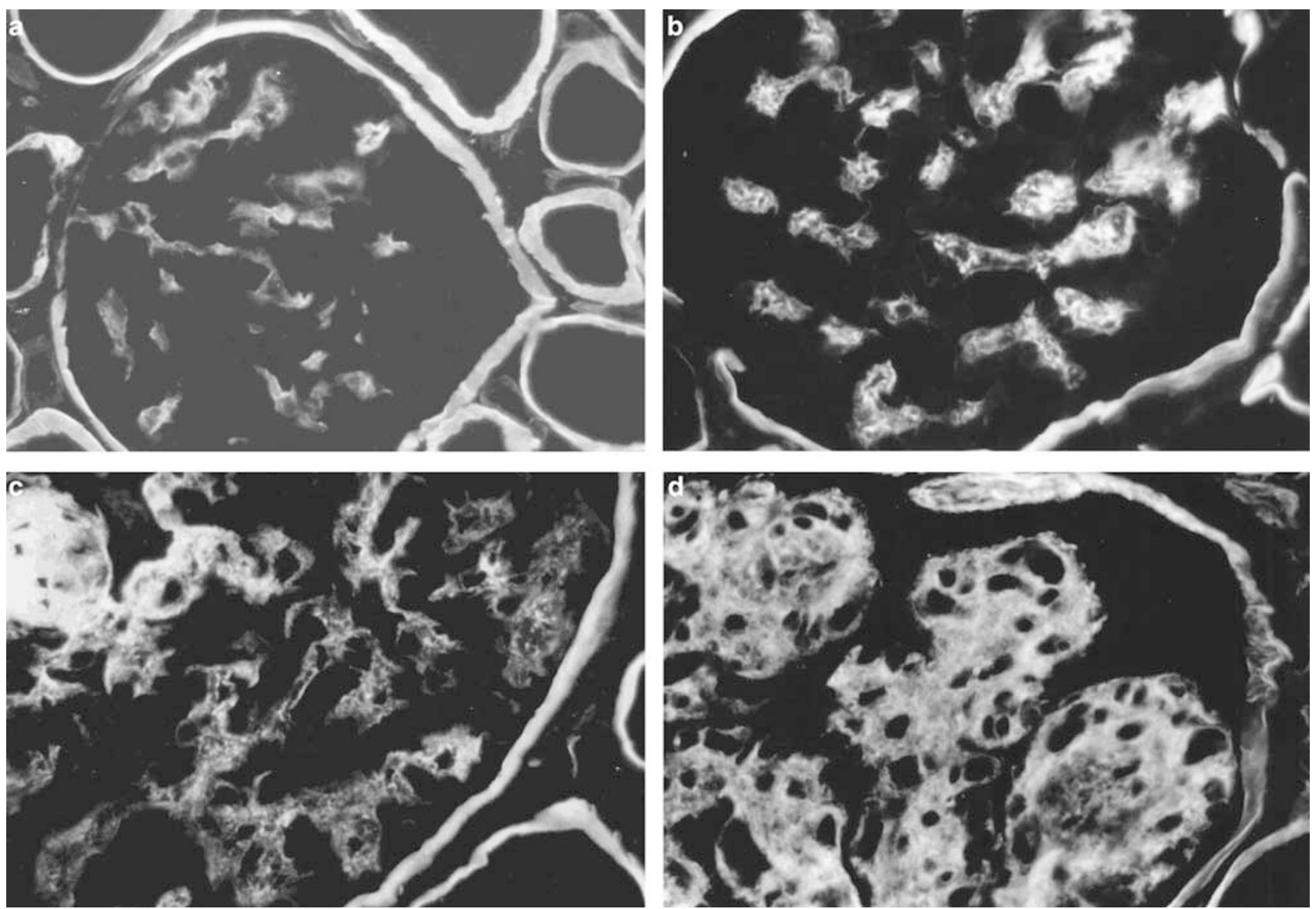

Figure 3 Immunofluorescence microscopy of glomeruli immunoreacted for Lm $\beta 1$ chain $(\times 550)$. (a) Normal kidney; (b) mild diabetic nephropathy; (c) severe diabetic nephropathy; (d) membranoproliferative glomerulonephritis. Lm $\beta 1$ chain is detected in both the normal and expanded mesangium. 
and $\beta 1$ chains were absent (Table 2, Figures 1a-5a, Lm $\alpha 1$ not shown). Only $\alpha 2, \beta 1$ and $\gamma 1$ chains were present in the mesangium. The intensity and extent of mesangial immunoreactivity for laminin chains, in contrast to type IV collagen, were quite variable in different normal kidneys ranging from trace focal to intense diffuse labeling.

The thickened glomerular basement membrane in mild diabetic nephropathy showed increased reactivity (both the area and the intensity of the staining) only with antibodies to the normal laminin constituents of glomerular basement membrane, namely Lm $\alpha 5, \beta 2$ and $\gamma 1$ chains (Table 2, Figures $1 \mathrm{~b}-5 \mathrm{~b}$ ). Mild to moderate mesangial expansion in diabetic nephropathy, in contrast, was associated not only with the increased reactivity with antibodies to the normal laminin constituents of mesangial matrix, but also with the aberrant expression of normal glomerular basement membrane components, Lm $\alpha 5$ and $\beta 2$ chains (Table 2, Figures 1b-5b). As the mesangial expansion progressed further in diabetic nephropathy, there was a striking decrease or disappearance of $\mathrm{Lm} \alpha 2$ chain, whereas other laminin chains and type IV collagen [ $\alpha 1(\mathrm{IV}) \mathrm{NC}]$ continued to increase (Figures 1c-5c and 6-8). These findings are summarized in Table 2. In the 12 diabetic nephropathy cases where mesangial fraction of glomerular volume was determined, the reduction in $\alpha 2$ chain intensity was only noted when mesangial fraction of glomerular volume exceeded 0.45 (4 cases) and in the area of nodular glomerulosclerosis.

In membranous nephropathy, like in mild diabetic nephropathy, there was thickening of the glomerular basement membrane with increased reactivity of antibodies to the normal glomerular basement membrane constituents $\operatorname{Lm} \alpha 5, \beta 2$ and $\gamma 1$ chains (Table 2, figures not shown).

Cases of Henoch-Schönlein purpura/IgA nephropathy expressed increased normal mesangial constituents $\alpha 2, \beta 1$ and $\gamma 1$ chains and aberrantly expressed $\operatorname{Lm} \alpha 5$ and $\beta 2$ chains (Table 2 , figures not shown).

Cases of membranoproliferative glomerulonephritis behaved similar to severe diabetic nephropathy, with a decrease or disappearance of $\mathrm{Lm} \alpha 2$ chain while overexpressing normal mesangial constituents $\beta 1$ and $\gamma 1$ and type IV collagen [ $\alpha 1(\mathrm{IV}) \mathrm{NC}]$ and aberrantly expressing $\alpha 5$ and $\beta 2$ chains (Table 2, Figures 1d-5d and 9).
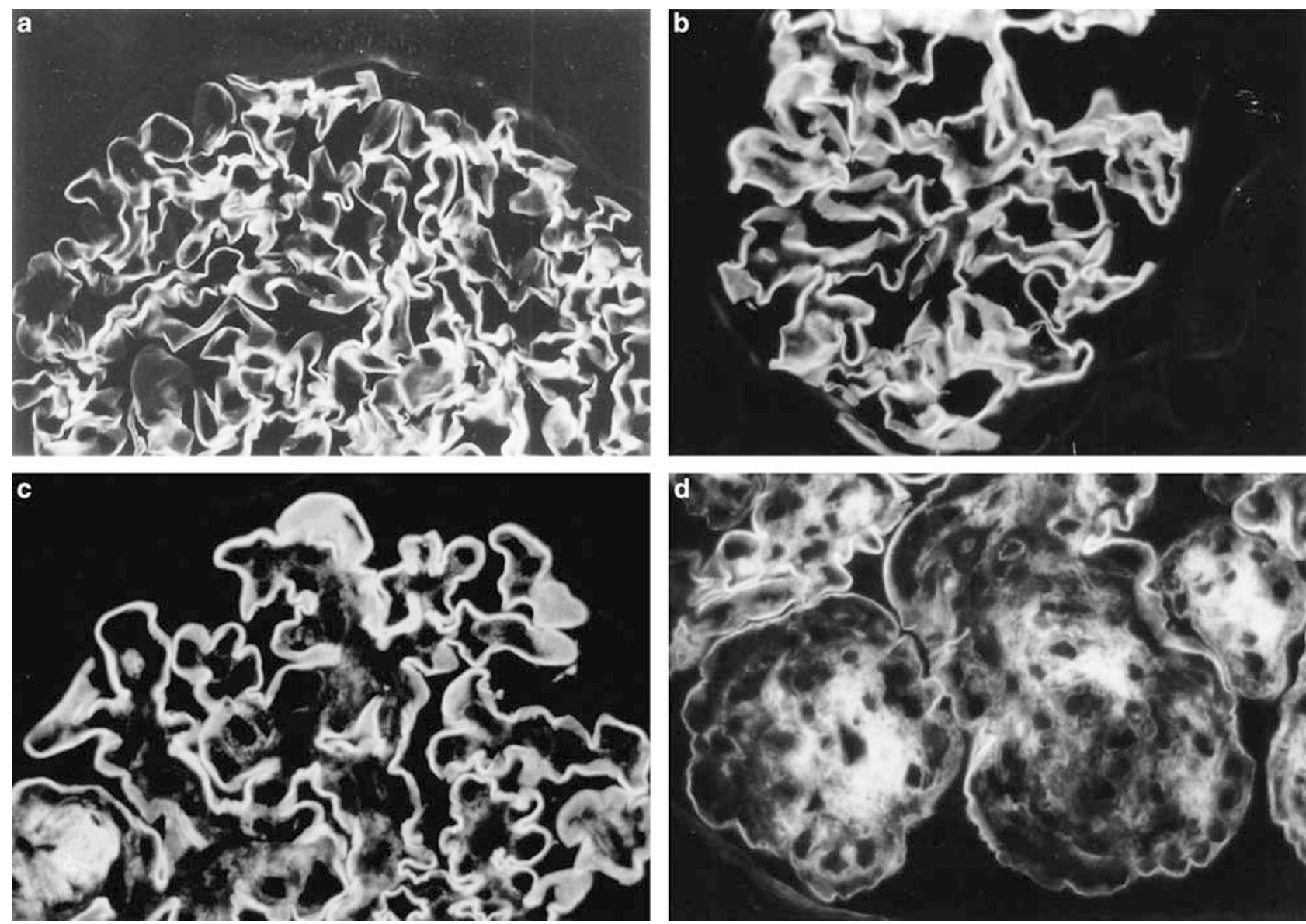

Figure 4 Immunofluorescence microscopy of glomeruli reacted for Lm $\beta 2$ chain $(\times 550)$. (a) Normal kidney; (b) mild diabetic nephropathy; (c) severe diabetic nephropathy; (d) membranoproliferative glomerulonephritis. The distribution and pattern of glomerular immunoreactivity are similar to those of $\mathrm{Lm} \alpha 5$ chain. 

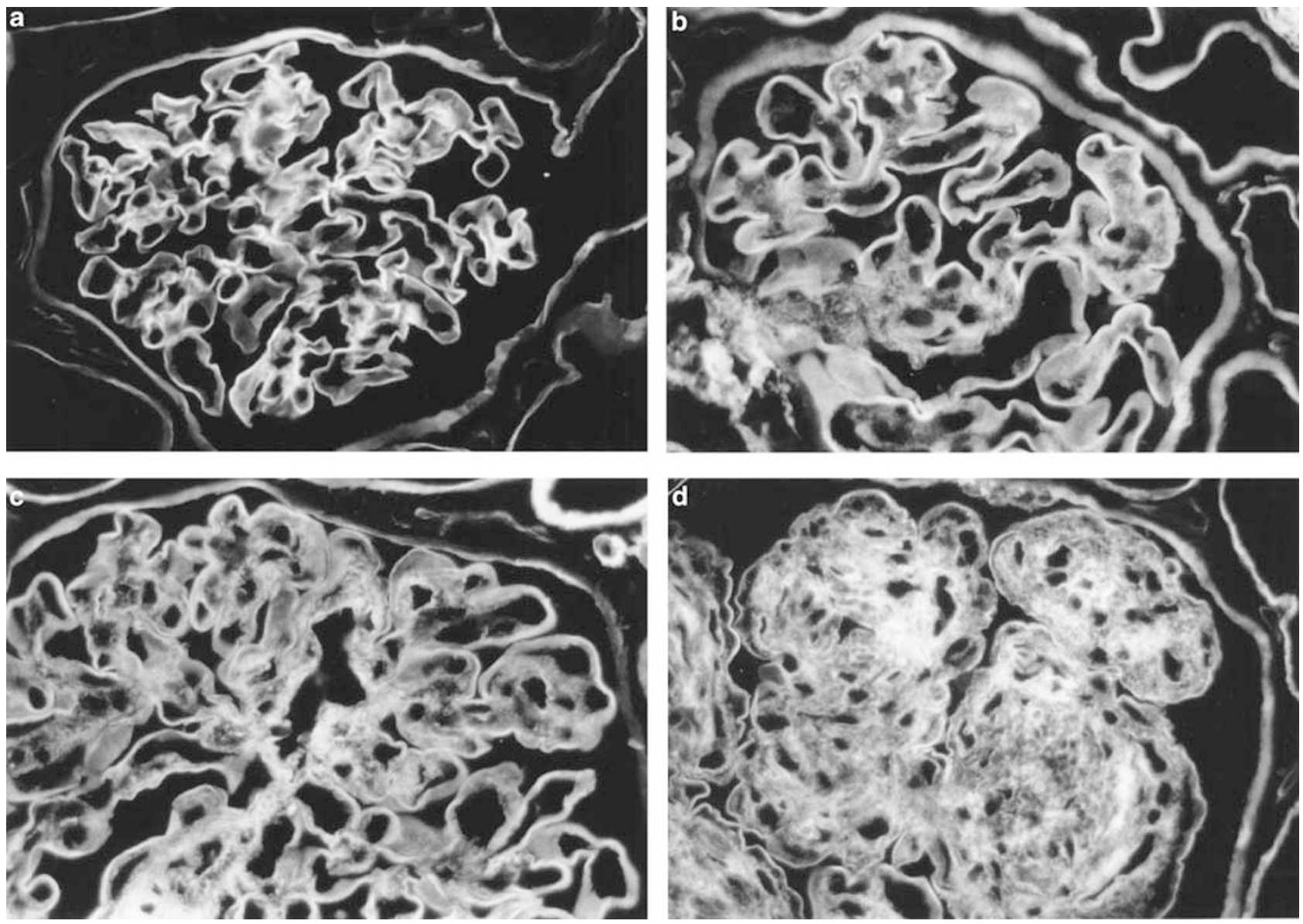

Figure 5 Immunofluorescence microscopy of glomeruli immunoreacted for Lm $\gamma 1$ chain $(\times 550)$. (a) Normal kidney; (b) mild diabetic nephropathy; (c) severe diabetic nephropathy; (d) membranoproliferative glomerulonephritis. In normal glomeruli, the Lm $\gamma 1$ chain overlies both the glomerular basement membrane and the mesangium. In diabetic nephropathy, there is intense immunoreactivity in both the thickened glomerular basement membrane and the expanded mesangium. In membranoproliferative glomerulonephritis, there is intense reactivity in the expanded mesangium with positive staining reaction in glomerular basement membrane.

\section{Discussion}

Glomerular structural-functional relationships have been demonstrated in a variety of kidney diseases. ${ }^{15-18}$ There is a close correlation between mesangial expansion and renal function in diabetic nephropathy and membranoproliferative glomerulonephritis. These structural changes are due, at least in part, to alterations in the composition of extracellular matrix and basement membrane constituents, which reflect disturbances in the balance between extracellular matrix and basement membrane synthesis and degradation. Although it is generally believed that these alterations involve only the normal extracellular matrix and basement membrane constituents, more recent studies have shown aberrant expression of basement membrane proteins in various renal diseases. In human Alport syndrome and in the murine collagen COL4A3 knockout model, type IV collagen $\alpha 1$ and $\alpha 2$ chains, type V collagen, type VI collagen and laminin $\alpha 2$ chains are abnormally expressed in the glomerular basement membrane in association with the absence of the $\alpha 3, \alpha 4$ and $\alpha 5$ chains of type IV collagen. ${ }^{8,9,19-22} \mathrm{Lm}-111(\alpha 1, \beta 1$ and $\gamma 1)$, normally present only in the mesangium was detected in the glomerular basement membrane of sclerotic glomeruli in murine lupus nephritis. ${ }^{23}$ Studies of membranous nephropathy have demonstrated increased expression in the glomerular basement membrane of normal constituents $\operatorname{Lm} \beta 2$ and $\alpha 1, \alpha 3$ and $\alpha 5$ chains of type IV collagen and variable aberrant expression of $\operatorname{Lm} \beta 1 .^{24}$

In our study, progressive mesangial expansion in diabetic nephropathy, membranoproliferative glomerulonephritis and Henoch-Schönlein purpura/IgA nephropathy was associated with aberrant mesangial expression of $\operatorname{Lm} \alpha 5$ and $\beta 2$ chains normally expressed only in adult glomerular basement membrane and with the decrease and the disappearance of mesangium-specific laminin chains, $\alpha 2$ in marked mesangial expansion. This loss of normally expressed and aberrant expression of Lm isoforms may be deleterious for renal function. Earlier studies by Kikkawa et $a l^{25}$ have demonstrated that mesangial cells organize the glomerular capillaries by adhering to a specific 

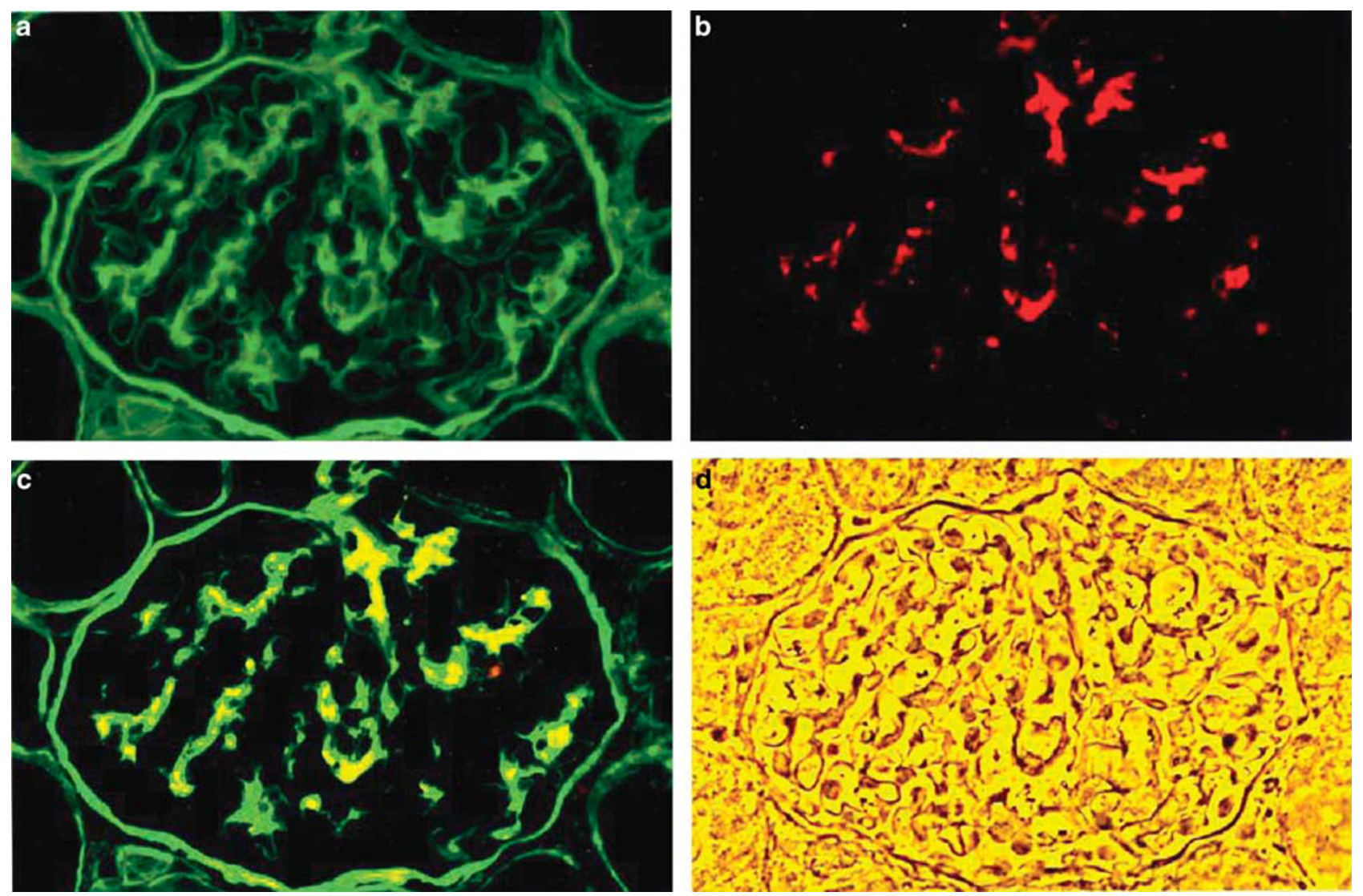

Figure 6 Dual fluorochrome staining of a normal glomerulus for (a) type IV collagen (FITC) and (b) Lm $\alpha 2$ chain (RITC) ( $\times 550$ ). (a) Both the glomerular basement membrane and the mesangium react for type IV collagen; (b) diffuse staining of the mesangium for Lm $\alpha 2$ chain; (c) when viewed under a filter system that permits visualization of both fluorochromes, there is an overlap (green and red to yellow/ orange) in the mesangial localization of type IV collagen and Lm $\alpha 2$ chain. (d) The same glomerulus is visualized by phase-contrast microscopy.

domain of $\operatorname{Lm} \alpha 5$ chain in the glomerular basement membrane. Thus, a disorganized laminin 521 with abnormal expression of glomerular basement membrane can likely lead to disorganization of glomerular capillaries and therefore to aberrant glomerular function.

Phenotypic alterations of extracellular matrix synthetic profiles of glomerular cells have been observed during nephrogenesis and in vitro studies. Both laminin $\alpha 5$ and $\beta 2$ chains are expressed in the mesangium of early and late capillary stages of fetal glomeruli with the disappearance in mesangium of normal adult kidneys (personal observation). Mesangial cells in vitro synthesize significant amount of interstitial collagens, which are not present in normal mesangium, most likely reflecting dedifferentiation. ${ }^{26}$ One of the explanations for the disappearance of laminin $\beta 2$ in the markedly expanded mesangium could be due to the masking of an epitope occurring due to the progression of mesangial expansion. However, since the two monoclonal antibodies we used were against different epitopes of laminin $\beta 2$ and both gave identical staining characteristics; the possibility of a 'hidden epitope' is negated. In addition, persistent expression of other laminin chains and type IV collagen in markedly expanded mesangium makes the hidden epitope explanation unlikely. It has been suggested that the mesangium in disease states may be populated by cells originating from extraglomerular sites such as the bone marrow or the juxtaglomerular apparatus. ${ }^{27-29}$ These translocated cells could express different matrix synthetic profiles, although it is equally plausible that resident mesangial cells undergo dedifferentiation due to disease related alterations in cytokines/growth factors or local architectural changes representing advancing disease processes.

We believe that changes in the A2 isoform of laminin reflect the degree of mesangial matrix expansion. In other words, in early/mild mesangial expansion as occurs in mild diabetic nephropathy or Henoch-Schönlein purpura/IgA nephropathy, there is an increase in expression of the A2 isoform, whereas in severe diabetic nephropathy or membranoproliferative glomerulonephritis, there is a decrease. Although it is unclear as to why this happens, we speculate that this may be due to masking of an epitope for the A2 isoform of laminin or dedifferentiation of mesangial cells, which occurs as mesangial expansion progresses.

In summary, the alterations in basement membrane composition in various renal diseases seem to 

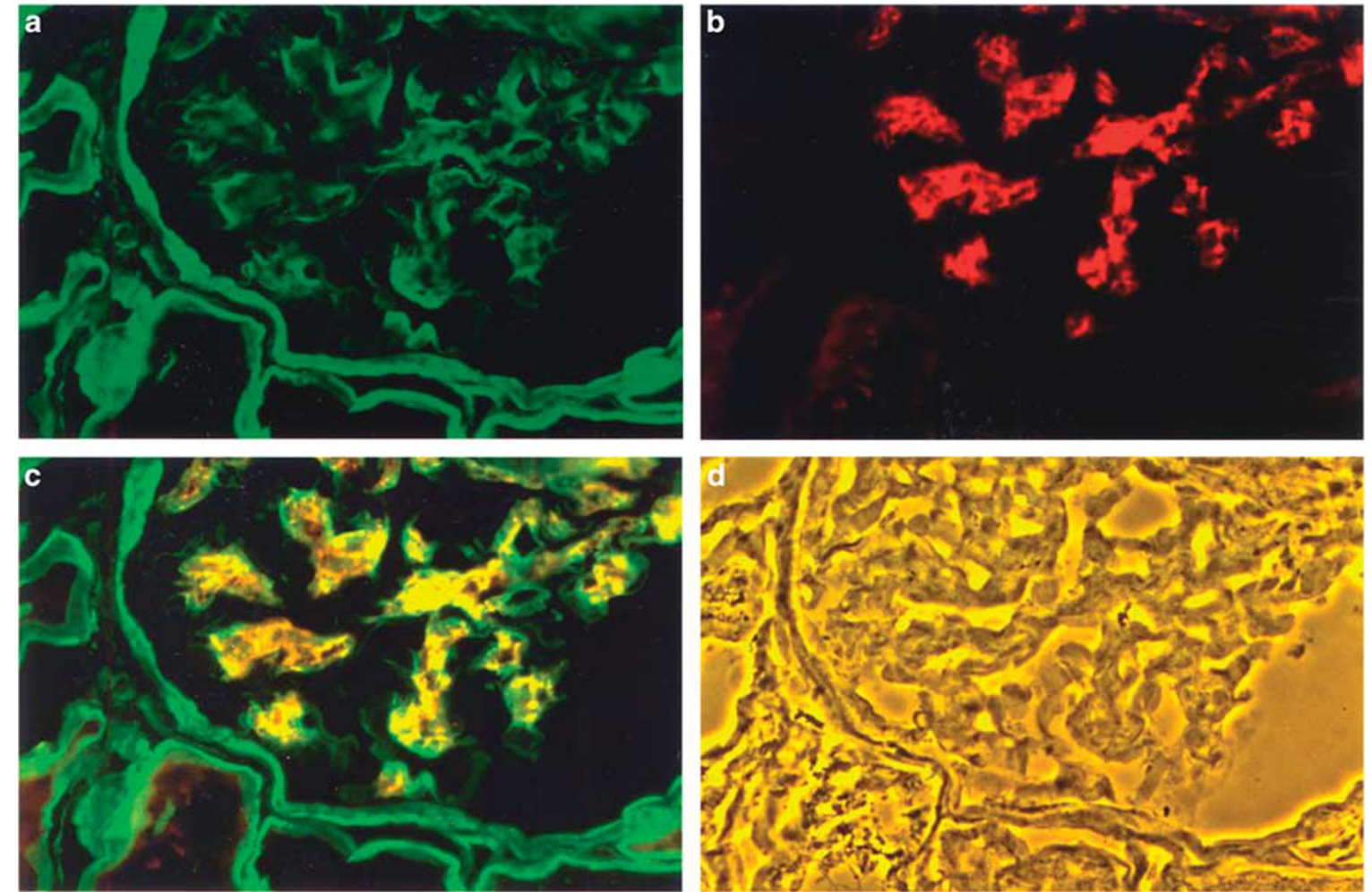

Figure 7 Dual fluorochrome studies of a glomerulus from mild diabetic nephropathy stained for (a) type IV collagen (FITC) and (b) Lm $\alpha 2$ chain $($ RITC) $(\times 550)$. (c) Note the increased and complete overlap of mesangial immunoreactivity for type IV collagen and Lm $\alpha 2$ chain. (d) The same glomerulus is visualized by phase-contrast microscopy.
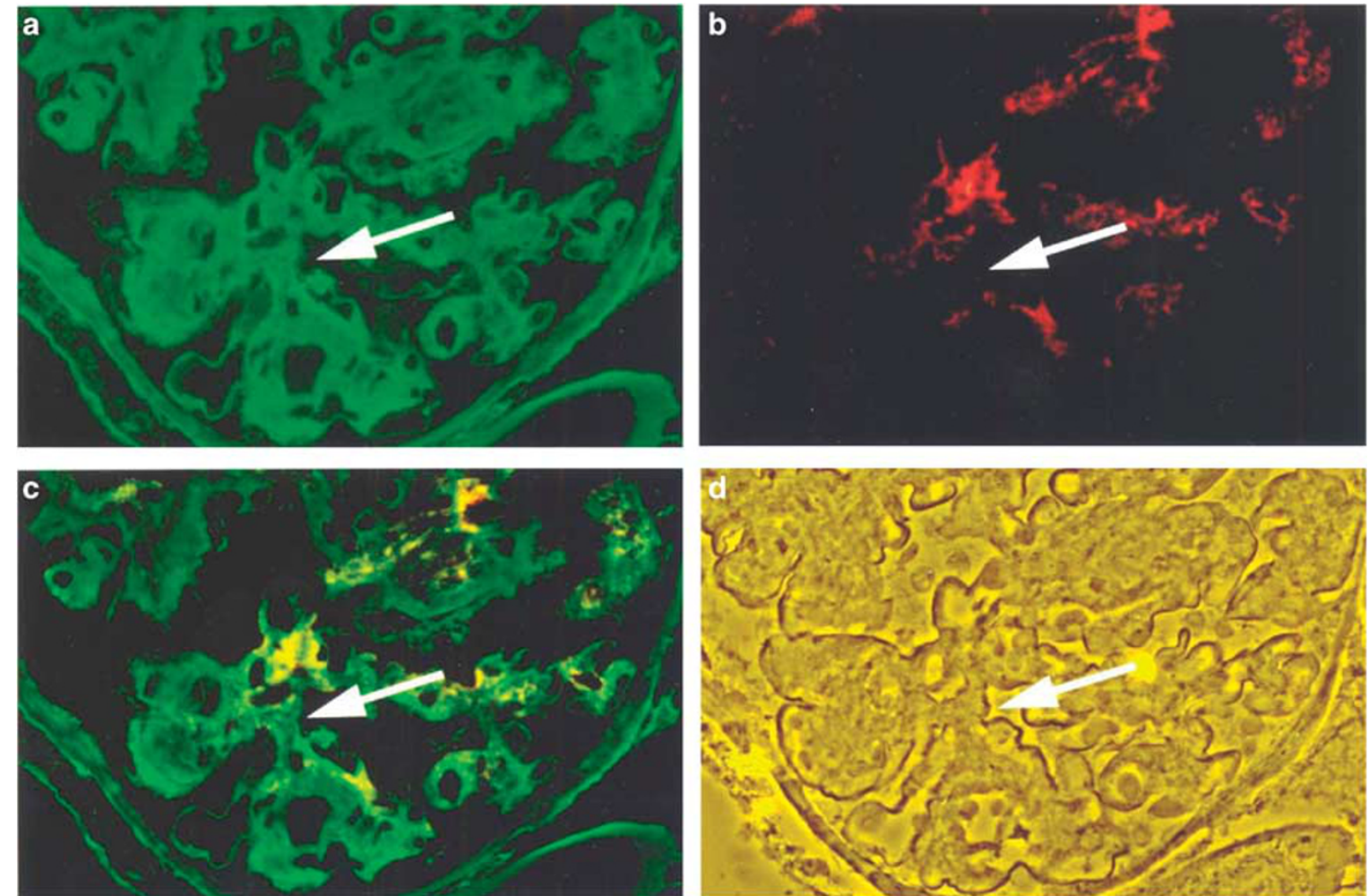

Figure 8 Dual fluorochrome studies of a glomerulus from severe diabetic nephropathy stained for (a) type IV collagen (FITC) and (b) Lm $\alpha 2$ chain (RITC) $(\times 550)$. While there is a diffuse increased mesangial staining for type IV collagen with glomerular basement membrane thickening, note the striking decrease to disappearance (arrow) of the $\mathrm{Lm} \alpha 2$ chain in the mesangium. (c) When both fluorochromes are visualized together, the area of loss is seen in green. (d) The same glomerulus is visualized by phase-contrast microscopy. 

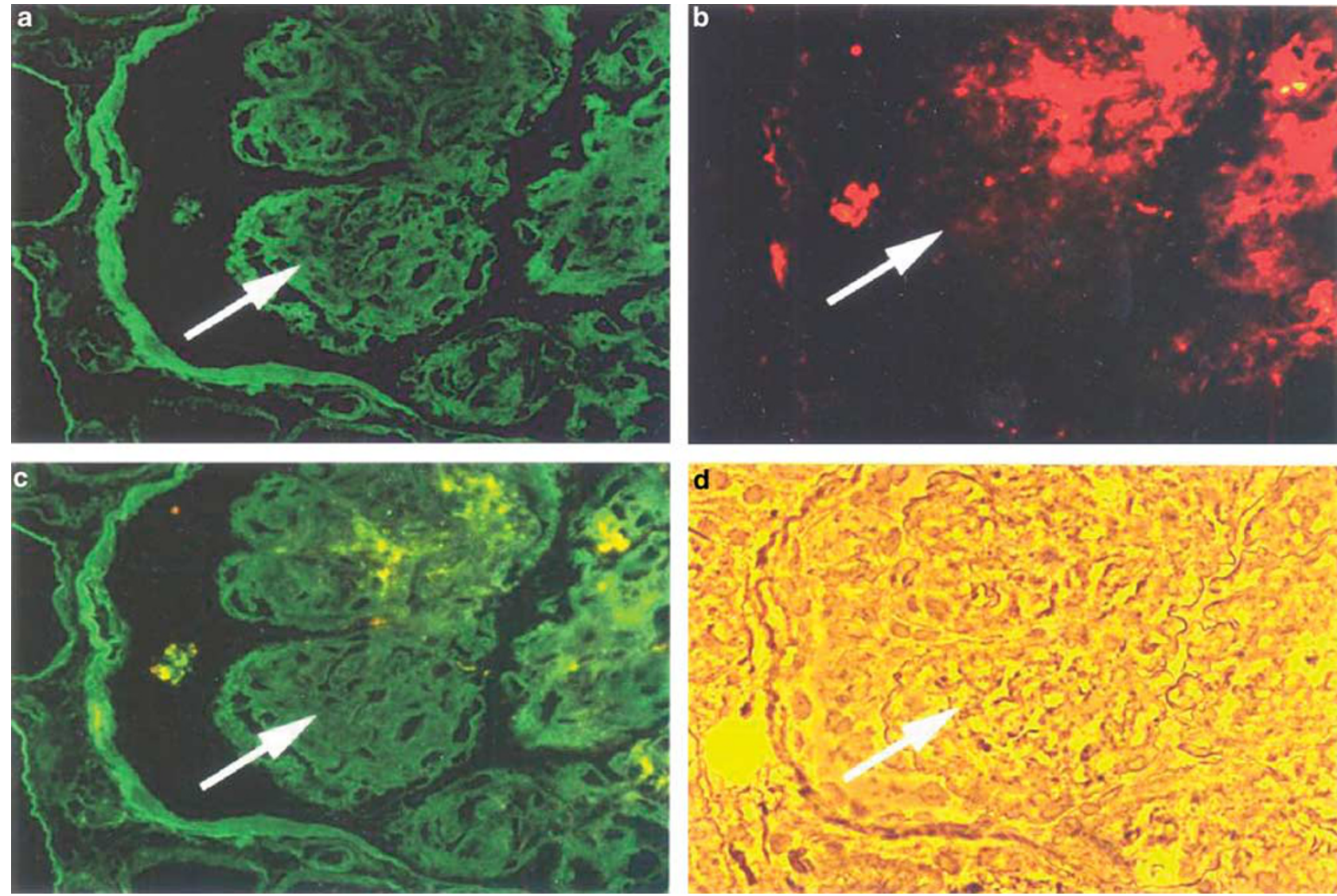

Figure 9 Dual fluorochrome studies of a glomerulus from membranoproliferative glomerulonephritis stained for (a) type IV collagen (FITC) and (b) Lm $\alpha 2$ chain (RITC) ( $\times 550)$. (c) As in severe diabetic nephropathy, there is a decrease to disappearance (arrow) of the Lm $\alpha 2$ chain in the mesangium, while diffuse increased mesangial immunoreactivity for type IV collagen persists. (d) The same glomerulus is visualized by phase-contrast microscopy.

not only reflect the balance between synthesis and degradation of normal basement membrane constituents, but also the aberrant new expression of basement membrane molecules. Thus, tissue remodeling may incorporate both an increase of normal constituents and emergence of abnormal constituents during disease exemplified by diabetes.

\section{Acknowledgement}

We thank Kathy Divine and Kim Pinkham for immunohistochemical staining.

\section{Disclosures/conflict of interest}

The authors declare no conflict of interest.

\section{References}

1 Aumailley M, Bruckner-Tuderman L, Carter WG, et al. A simplified laminin nomenclature. Matrix Biol 2005;24:326-332.

2 Miner JH. Building the glomerulus: a matricentric view. J Am Soc Nephrol 2005;16:857-861.
3 Miner JH, Yurchenco PD. Laminin functions in tissue morphogenesis. Annu Rev Cell Dev Biol 2004;20: 255-284.

4 Timpl R, Brown JC. The laminins. Matrix Biol 1994;14:275-281.

5 Colognato H, Yurchenco PD. Form and function: the laminin family of heterotrimers. Dev Dyn 2000; 218:213-234.

6 Virtanen I, Laitinen L, Korhonen M. Differential expression of laminin polypeptides in developing and adult human kidney. J Histochem Cytochem 1995;43: 621-628.

7 Virtanen I, Gullberg D, Rissanen J, et al. Laminin alpha1-chain shows a restricted distribution in epithelial basement membranes of fetal and adult human tissues. Exp Cell Res 2000;257:298-309.

8 Kashtan CE, Kim Y, Lees GE, et al. Abnormal glomerular basement membrane laminins in murine, canine, and human Alport syndrome: aberrant laminin alpha2 deposition is species independent. J Am Soc Nephrol 2001;12:252-260.

9 Petajaniemi N, Korhonen M, Kortesmaa J, et al. Localization of laminin alpha4-chain in developing and adult human tissues. J Histochem Cytochem 2002; 50:1113-1130.

10 Miner JH, Sanes JR. Collagen IV alpha 3, alpha 4, and alpha 5 chains in rodent basal laminae: sequence, distribution, association with laminins, and developmental switches. J Cell Biol 1994;127:879-891. 
11 Miner JH, Sanes JR. Molecular and functional defects in kidneys of mice lacking collagen alpha 3(IV): implications for Alport syndrome. J Cell Biol 1996;135:1403-1413.

12 Noakes PG, Miner JH, Gautam M, et al. The renal glomerulus of mice lacking s-laminin/laminin beta 2: nephrosis despite molecular compensation by laminin beta 1 . Nat Genet 1995;10:400-406.

13 Jarad G, Cunningham J, Shaw AS, et al. Proteinuria precedes podocyte abnormalities in Lamb2-/- mice, implicating the glomerular basement membrane as an albumin barrier. J Clin Invest 2006;116:2272-2279.

14 Kim Y, Butkowski R, Burke B, et al. Differential expression of basement membrane collagen in membranous nephropathy. Am J Pathol 1991;139:1381-1388.

15 Hattori M, Kim Y, Steffes MW, et al. Structuralfunctional relationships in type I mesangiocapillary glomerulonephritis. Kidney Int 1993;43:381-386.

$16 \mathrm{Kim} \mathrm{KH,} \mathrm{Kim} \mathrm{Y,} \mathrm{Gubler} \mathrm{MC,} \mathrm{et} \mathrm{al.} \mathrm{Structural-}$ functional relationships in Alport syndrome. J Am Soc Nephrol 1995;5:1659-1668.

17 Caramori ML, Kim Y, Huang C, et al. Cellular basis of diabetic nephropathy: 1. Study design and renal structural-functional relationships in patients with longstanding type 1 diabetes. Diabetes 2002;51:506-513.

18 Mauer SM, Steffes MW, Ellis EN, et al. Structuralfunctional relationships in diabetic nephropathy. J Clin Invest 1984;74:1143-1155.

19 Kashtan CE, Kim Y. Distribution of the alpha 1 and alpha 2 chains of collagen IV and of collagens $\mathrm{V}$ and VI in Alport syndrome. Kidney Int 1992;42:115-126.

20 Cosgrove D, Rodgers $\mathrm{K}$, Meehan $\mathrm{D}$, et al. Integrin alpha1beta1 and transforming growth factor-beta1 play distinct roles in alport glomerular pathogenesis and serve as dual targets for metabolic therapy. Am J Pathol 2000;157:1649-1659.

21 Hamano Y, Grunkemeyer JA, Sudhakar A, et al. Determinants of vascular permeability in the kidney glomerulus. J Biol Chem 2002;277:31154-31162.

22 Abrahamson DR, Prettyman AC, Robert B, et al. Laminin1 reexpression in Alport mouse glomerular basement membranes. Kidney Int 2003;63:826-834.
23 Kootstra CJ, Bergijk EC, Veninga A, et al. Qualitative alterations in laminin expression in experimental lupus nephritis. Am J Pathol 1995;147:476-488.

24 Fischer E, Mougenot B, Callard P, et al. Abnormal expression of glomerular basement membrane laminins in membranous glomerulonephritis. Nephrol Dial Transplant 2000;15:1956-1964.

25 Kikkawa Y, Virtanen I, Miner JH. Mesangial cells organize the glomerular capillaries by adhering to the $\mathrm{G}$ domain of laminin alpha5 in the glomerular basement membrane. J Cell Biol 2003;161: 187-196.

26 Haralson MA, Jacobson HR, Hoover RL. Collagen polymorphism in cultured rat kidney mesangial cells. Lab Invest 1987;57:513-523.

27 Ito T, Suzuki A, Imai E, et al. Bone marrow is a reservoir of repopulating mesangial cells during glomerular remodeling. J Am Soc Nephrol 2001;12:2625-2635.

28 Cornacchia F, Fornoni A, Plati AR, et al. Glomerulosclerosis is transmitted by bone marrow-derived mesangial cell progenitors. J Clin Invest 2001;108: 1649-1656.

29 Hugo C, Shankland SJ, Bowen-Pope DF, et al. Extraglomerular origin of the mesangial cell after injury. A new role of the juxtaglomerular apparatus. J Clin Invest 1997;100:786-794.

30 Leivo I, Engvall E. Merosin, a protein specific for basement membranes of Schwann cells, striated muscle, and trophoblast, is expressed late in nerve and muscle development. Proc Natl Acad Sci USA 1988;85:1544-1548.

31 Engvall E, Davis GE, Dickerson K, et al. Mapping of domains in human laminin using monoclonal antibodies: localization of the neurite-promoting site. J Cell Biol 1986;103:2457-2465.

32 Sanes JR, Chiu AY. The basal lamina of the neuromuscular junction. Cold Spring Harb Symp Quant Biol 1983;48 (Part 2):667-678.

33 Butkowski RJ, Wieslander J, Kleppel M, et al. Basement membrane collagen in the kidney: regional localization of novel chains related to collagen IV. Kidney Int 1989;35:1195-202. 